\title{
A Cooperative Spectrum Sensing Scheme Based on Extension Theory for Cognitive Radio Sensor Networks
}

\author{
Sheng Ouyang ${ }^{1}$, Pin Wan ${ }^{1}$, Yonghua Wang ${ }^{1,2,3, *}$, Liyuan Wang ${ }^{1}$ and Qinruo Wang ${ }^{1}$ \\ ${ }^{I}$ School of Automation, Guangdong University of Technology, Guangdong, 510006, China \\ ${ }^{2}$ School of Electronic and Information Engineering, South China University of Technology, Guangzhou, 510641, China \\ ${ }^{3}$ Shenzhen Key Laboratory of High Performance Data Mining, Shenzhen, 518055, China
}

\begin{abstract}
This paper proposes a cooperative censoring spectrum sensing scheme based on dependent function of extension theory for Cognitive Radio Sensor Networks (CRSN). The scheme uses the dependent function of Extension theory to identify the presence or absence of the licensed user's (LU) signal, and then calculate the related degree through dependent function to identify the initial test results of licensed users, and then send these results to the fusion center. Use a trust evaluation scheme based on noise jamming and channel attenuation for each node, and then this trust evaluation result of each node is sent to the fusion center. The fusion center makes the final decision by the K-M rule. Simulation results show that the proposed scheme could improve the detect probability effectively.
\end{abstract}

Keywords: Cooperative Spectrum Sensing, CRSN, Data Fusion, Dependent Function, Extension Theory.

\section{INTRODUCTION}

Today, wireless sensor networks (WSN) are used in many applications, including military, public security, public health, intelligent transportation, intelligent household, environmental monitoring, etc [1-3]. With the increasing need of wireless communication to radio frequency spectrum, the existing frequency spectrum can't meet the target required. Cognitive radio [4] can achieve higher spectrum efficiency by dynamic spectrum access. Cognitive Radio Sensor Networks (CRSN) be formed by applying cognitive radio to the WSN. So far, the spectrum sensing techniques [5-7] fall into three categories: Matched filter detection [8], Cyclostationary feature detection [9], and Energy detection [10]. Among them, the energy detector is the most convenient and the most accurate. To solve the problem of potential interference to the LU, cooperative spectrum sensing schemes have been proposed to take advantage of the spatial diversity in wireless [11]. In [12], energy detection for cooperative spectrum sensing has been proposed to save energy. A censor-based cooperative spectrum sensing scheme using fuzzy logic for cognitive radio sensor networks is proposed in [13]. But in this scheme, the CRSN nodes are assumed to be trustworthy, and existence of the LU's signal cannot be expressed in digits. Inspired by dependent function of extension theory, a new spectrum sensing scheme based on dependent function has been presented in this paper.
In this paper, based on the definitions of the extensible set and dependent function [14], a method under extension transformation is studied. The researching objects of extenics are contradictory problems. And extenics is a breakthrough of the weakness that conventional mathematics can only solve the precise problem. Seeking solution of failing to detect and enhancing precision a new angle. Extensive detecting technology researches the conformation of detecting strategy and explores inscrutable and securable matter elements converting principle by extension reasoning, avoiding the aimlessness and uncertainness of detecting. And then a set of advanced detecting theories that differ from conventional methods are established [15].

In this method, the presence and absence of the LU's signal are transformed to the interval value. Every node, through the dependent function, is sent to the fusion center. And the reliability of each CRSN node is different. So the fusion center makes the final decision according to the local decisions and the reliability of each CRSN node. This way can't only improve the efficiency of test, but also save the energy.

\section{ENERGY DETECTION MODEL OF THE SYSTEM}

Energy detection is a sub-optional signal detection technique which has been extensively used in radiometry. Cognitive user can judge the working status of the licensed user (LU) by the detection theory of two hypotheses.

$\left\{\begin{array}{l}H_{1}: x(t)=h(t) \cdot s(t)+n(t) \\ H_{0}: x(t)=n(t)\end{array}\right.$

Where $H_{0}$ and $H_{1}$ respectively mean that the LU's signal is absence and presence. $x(t)$ represents that the signal 


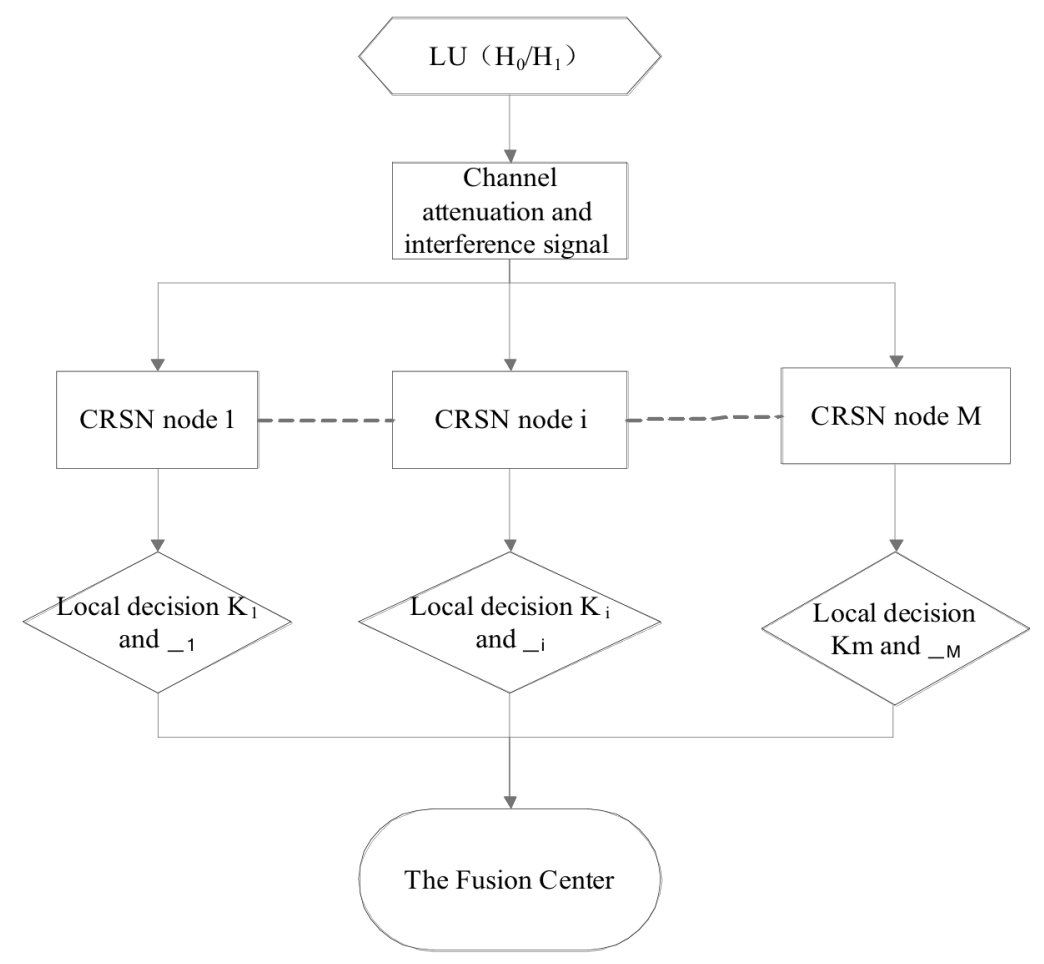

Fig. (1). Proposed spectrum sensing scheme.

received by the CRSN node, $s(t)$ represents that the signal transmitted by the licensed user, $h(t)$ denotes the amplitude gain of the channel and $n(t)$ is additive the white Gaussian noise [16].

To implement energy detection, a band pass filter is used to obtain a signal in the desired frequency band. Then the measured energy of received signal is calculated according to the energy of received signal's samples:

$E=\sum_{t=0}^{W-1}|x(t)|^{2}$

Where $W$ is the number of samples: $W=2 T B$, where $\mathrm{B}$ and $\mathrm{T}$ are signal bandwidth and correspondent to detection time, respectively [10].

Without loss of generality, we assume that the noise at each sample is a Gaussian random variable with zero mean and unit power. When the LU doesn't send out signal, the distribution of energy decision statistic is Central chisquared distribution. When the LU sends out signal, the distribution of energy decision statistic is noncentral chisquared distribution [17]. When $W$ is relatively large (e.g. $W>200$ ), the energy $E$ can be close to a Gaussian random variable [10].

$E \sim\left\{\begin{array}{cc}N(W, 2 W) & H_{0} \\ N(W *(1+S N R), 2 W *(1+S N R)) & H_{1}\end{array}\right.$

Where $S N R$ is the signal noise ratio in the CRSN nodes. The Gaussian distribution is showed with $N\left(\mu, \sigma^{2}\right)$. The parameter $\mu$ is expectation, and the parameter $\sigma^{2}$ is variance. When LU doesn't send out signal, $\mu$ and $\sigma^{2}$ are equal to $W$ and $2 W$, respectively. When LU send out signal, $\mu$ and $\sigma^{2}$ are equal to $W *(1+S N R)$ and $2 W *(1+S N R)$, respectively.

\section{PROPOSED SPECTRUM SENSING SCHEME}

To detect the LU's status, we propose a spectrum sensing scheme. In Fig. (1), each CRSN node detects the LU's signal. But, there exist noise and channel attenuation. The signal of received should be analysis. According to the dependent functions of extension theory, we get the local decision $\mathrm{K}$ and $\varphi$. And then these local decisions are transmitted to the fusion center for the last decision.

To get the result of the local decision, we use the matterelement to describe the LU and the cognitive user (CU).

\subsection{Matter-element Describing}

We use an ordered triad:

$R=(T, c, v)$

The basic element for describing things is called matterelement, where $T$ represents the matter; $c$ is the characteristics; $v$ is the $T^{\prime} S$ measure about the characteristics $c$; the expression $v=c(T)$ describes the relation between quality and quantity. So we can describe the LU and the cognitive user by matter-element [14].

Goal matter-element:

$\mathrm{G}=\left(\mathrm{LU}\right.$, working condition, $H_{0}$ (the absence) $/ H_{1}$ (the presence)); 


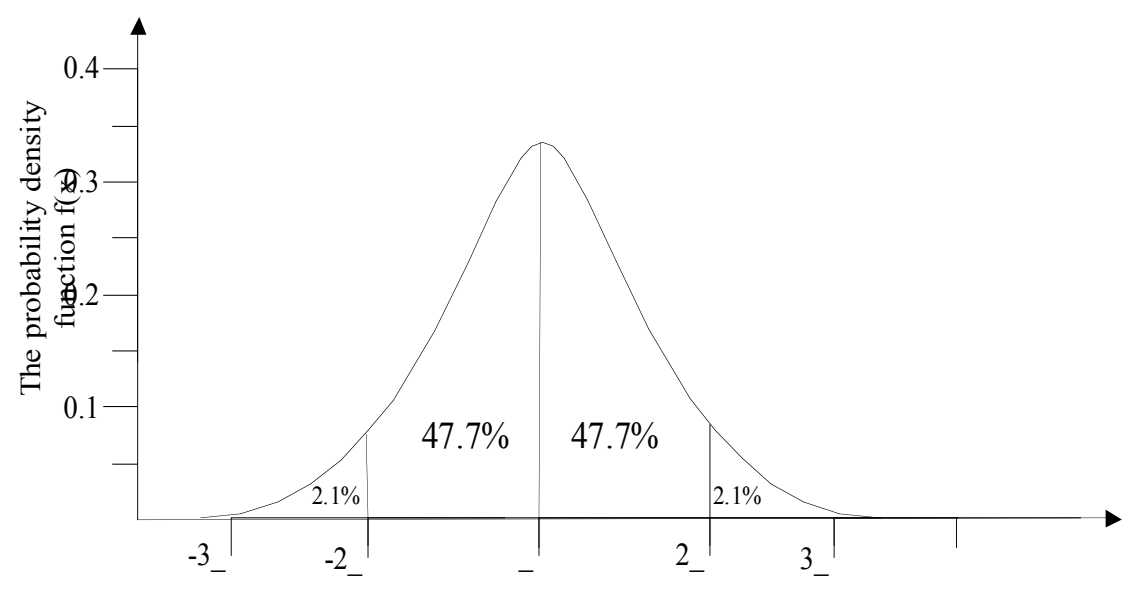

The random variable $\mathrm{x}$

Fig. (2). The probability density function of Gauss distribution.

Conditional matter-element:

$\mathrm{R}=(\mathrm{CU}$, receipt signal, $\mathrm{E})$;

Because the channel fading and noise signal have negative effects on sensing performance of CU. We must consider that each CRSN node has different $S N R$ and different distance away from LU.

$$
R=\left(\begin{array}{ccc}
\text { CRSN } & \text { receipt signal } & \mathrm{E}_{\mathrm{i}} \\
& \text { distance } & \mathrm{L}_{\mathrm{i}} \\
& \text { signal noise ratio } & \mathrm{SNR}_{\mathrm{i}}
\end{array}\right)
$$

Where $L_{i}$ is the distance between the $i$-th node and the LU, and $S N R_{i}$ is the signal noise ratio of $i$-th node, where $E_{i}$ is the energy of $i$-th node received signal $(i=1,2, \cdots, M)$.

\subsection{Matter-element Transformations}

Because the goal matter-element $G$ can't be directly judged by conditions of the matter-element $\mathrm{R}$, we should transform the goal matter-element. According to the formula (3), the $E$ can be described as a Gaussian random variable.

$$
\left\{\begin{array}{l}
H_{0} \sim N(W, 2 W) \\
H_{1} \sim N(W *(1+S N R), 2 W *(1+S N R))
\end{array}\right.
$$

The probable density function of Gauss distribution is described as following:

In Fig. (2), we can see that about $68.3 \%$ of values drawn from a normal distribution are within one standard deviations $\sigma$ away from the mean; about $95.4 \%$ of the values lie within two standard deviations; and about $99.7 \%$ are within there standard deviation. So the goal matter-element can be transformed to:

$$
G=\left(\begin{array}{ccc}
L U & H_{0} & (W-3 * \sqrt{2 W} W+3 * \sqrt{2 W}) \\
& H_{1} & \left(\begin{array}{l}
W *(1+S N R)-3 * \sqrt{2 W *(1+S N R)} \\
W *(1+S N R)+3 * \sqrt{2 W *(1+S N R)}
\end{array}\right)
\end{array}\right)
$$

\subsection{Dependent Function}

Dependent functions describe the degree of the property. When the LU's signal is absent $\left(H_{0}\right)$, the range of energy $E$ is $\left(x_{1}, y_{1}\right)$; When the LU's signal is present $\left(H_{1}\right)$, the range of energy $E$ is $\left(x_{2}, y_{2}\right)$. In order to calculate the dependent degree of $H_{1}$ and $H_{0}: \rho\left(H_{0}, H_{1}\right)$, we use the following formula:

$\rho\left(H_{0}, H_{1}\right)=\frac{1}{2}\left[\rho\left(x_{1},\left(x_{2}, y_{2}\right)\right)+\rho\left(y_{1},\left(x_{2}, y_{2}\right)\right)\right]$

Where $\rho\left(x_{1},\left(x_{2}, y_{2}\right)\right)$ and $\rho\left(y_{1},\left(x_{2}, y_{2}\right)\right)$ are dependent function. We can see that $\left(x_{2}, y_{2}\right)$ and $\left(x_{1}, y_{1}\right)$ are $(W *(1+S N R)-3 \sqrt{2 W *(1+S N R)}$,

$W *(1+S N R)+3 \sqrt{2 W *(1+S N R)})$

$(W-3 \sqrt{2 W}, W+3 * \sqrt{2 W})$ by the formula (7), respectively.

In this paper we use a simple dependent function as following:

$\rho= \begin{cases}\frac{x-x_{1}}{x_{0}-x_{1}} & x \leq x_{0} \\ \frac{y_{1}-x}{y_{1}-x_{0}} & x>x_{0}\end{cases}$

Where $x_{0}$ is the best value of range $\left(x_{1}, y_{1}\right)$.

According to the formulas (7), (8) and (9), we can calculate the relational value $\rho\left(H_{0}, H_{1}\right)$. We know that $\rho\left(H_{0}, H_{1}\right)$ is changed as the $S N R$ changed, when $W$ is invariant. And the interval of $H_{1}$ and $H_{0}$ is decreased as the $S N R$ decrease. So the critical value exists when the interval of $H_{1}$ and the interval of $H_{0}$ intersect with each other. In Fig. (3), we can see that the critical value is $\beta$.

We can work out the critical value $\beta=-(3 * \sqrt{2 W})$ by the formula (7), (8) and (9). Then we do the following analysis: 


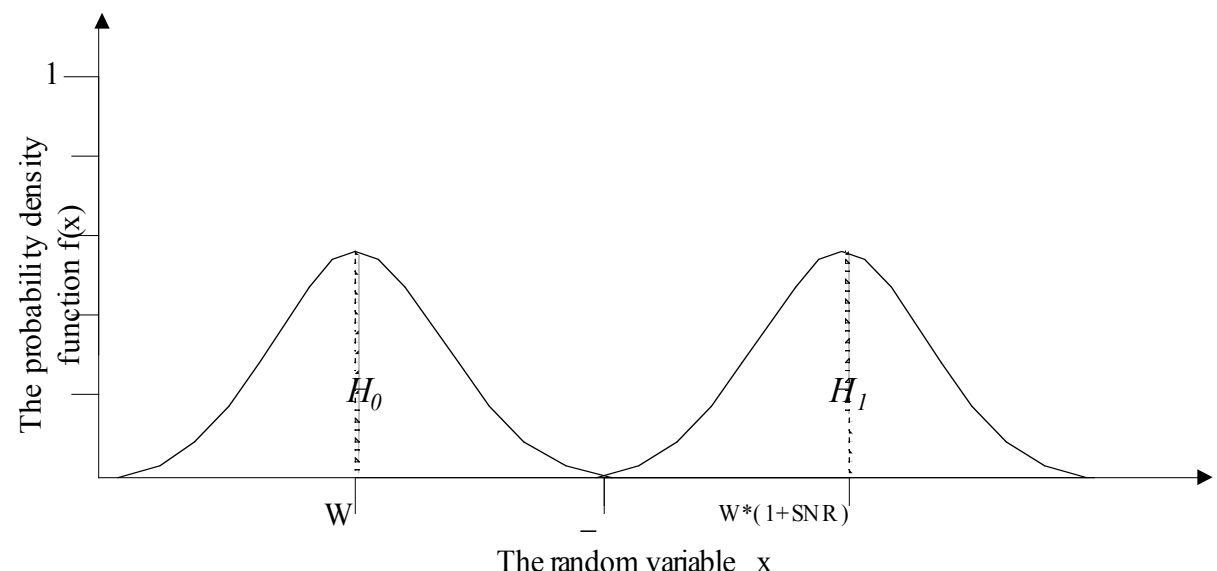

Fig. (3). The probable density function of $H_{0}$ and $H_{1}$.

When $\rho\left(H_{0}, H_{1}\right)>\beta$, the two sections of $H_{0}$ and $H_{1}$ are away from each other. When the energy of cognitive user (CU) received belong to $H_{0}$ or $H_{1}$, the LU should be $H_{0}$ or $H_{1}$, respectively. So when the $\mathrm{CU}$ receives energy value $E \in H_{0}$, output $k=-1$; when the $\mathrm{CU}$ receives energy value $E \in H_{1}$, output $k=1$.

When $\rho\left(H_{0}, H_{1}\right)<\beta$, the two sections of $H_{0}$ and $H_{1}$ are very close. When $E$ is the one value between $H_{0}$ and $H_{1}$, it is difficult to determine the working state of LU. Then we should use dependent functions to calculate relational degree of $E$ based on $H_{0}$ and $H_{1}: \rho\left(E, H_{1}\right)$ and $\rho\left(E, H_{0}\right)$.

According to the formula (7) and (9):

$$
\begin{gathered}
\rho\left(E, H_{0}\right)=\frac{(W+3 * \sqrt{2 W})-E}{3 * \sqrt{2 W}} \quad(E \geq W) \\
\rho\left(E, H_{1}\right)=\frac{E-(W(1+S N R)-3 * \sqrt{2 W(1+S N R)})}{3 * \sqrt{2 W(1+S N R)}} \\
(E \leq W(1+S N R))
\end{gathered}
$$

In order to determine the final decision, we need to make a calculation as following:

$$
K=\frac{\rho\left(E, H_{1}\right)-\rho\left(E, H_{0}\right)}{\rho\left(E, H_{1}\right)+\rho\left(E, H_{0}\right)}
$$

When $K<-a$, the state of the LU is $H_{0}$; when $K>a$, the state of the LU is $H_{1}$. The decision $K$ is transmitted to the fusion center only if $K<-a$ or $K>a$, where $a$ is the censoring threshold, $a \in(0,1)$.

\subsection{Reliability Calculation}

The distance is different between each CRSN nodes and $\mathrm{LU}$, and the effect of the interference signal is different for each CRSN nodes [18]. Consequently, the SNR parameter and the distance parameter should be transmitted to the fusion center.

The distance parameter of one CRSN node is $L_{i}$ $(i=1,2, \cdots, M)$. We assume that $L_{\max }$ is the maximum value, $L_{\min }$ is the minimum value. Because the CRSN node is more near to the $\mathrm{LU}$, the decision is more accurate. The best value is $L_{\min }$. In the same way, $S N R_{i}$ is the signal noise ratio of one node. $S N R_{\max }$ is the maximum value of $S N R_{i}, S N R_{\text {min }}$ is the minimum value of $S N R_{i}$. According to the formula of (9), we can calculate the reliability of each CRSN node:

$$
\rho\left(L_{i}\right)=\frac{L_{i}-L_{\max }}{L_{\min }-L_{\max }}
$$

$\rho\left(S N R_{i}\right)=\frac{S N R_{i}-S N R_{\text {min }}}{S N R_{\text {max }}-S N R_{\text {min }}}$

The final decision is under the influence of interference signal and channel fading, we should consider $\rho(L)$ and $\rho(S N R)$. We assume that distance-weighted is $\omega_{L}=0.5$, and weighting of SNR is $\omega_{S N R}=0.5$. The final influence degree is $\varphi$ of one node:

$\varphi_{i}=\omega_{L} * \rho\left(L_{i}\right)+\omega_{S N R} * \rho\left(S N R_{i}\right)$

Then the credibility $\varphi_{i}$ is transmitted to the fusion center.

\subsection{Data fusion at the Fusion Center}

The fusion center receives the first local decisions $K_{i}$ and the credibility $\varphi_{i}$ from the CRSN nodes. The decision of reliability of each CRSN nodes is calculated as follows:

$u_{i}=K_{i} * \phi_{i}$

The summarize is $U$, then send it to fusion center.

$U=\sum_{i=1}^{M} u_{i}$ 


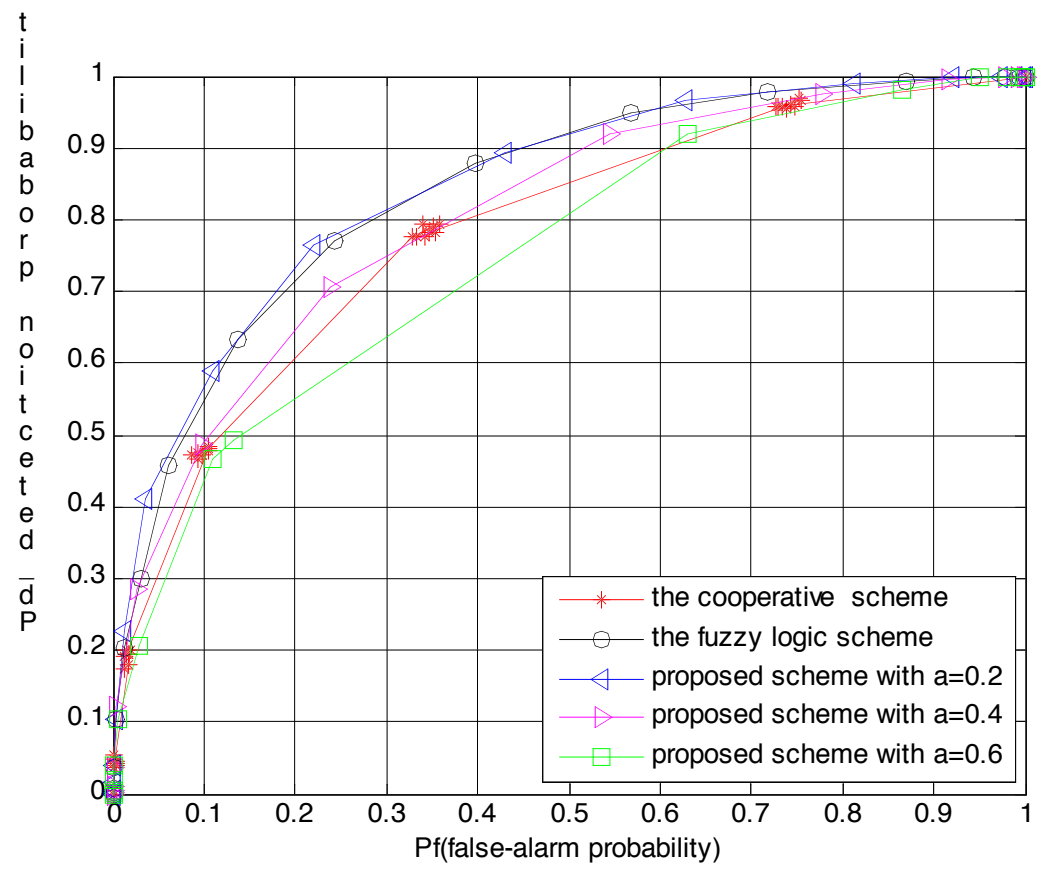

Fig. (4). The ROC curves of our proposed scheme vs. the cooperative scheme and the fuzzy logic scheme. And the SNRs of LU's signal at nodes are $-15,-14,-13,-12,-11$ and $-14 \mathrm{~dB}$, respectively.

The final decision is made by applying the $\mathrm{K}-\mathrm{M}$ rule:

$H=\left\{\begin{array}{cc}H_{1} & U>0 \\ H_{0} & \text { otherwise }\end{array}\right.$

By substituting (16) and (17) into (18), we have the final decision fusion rule as follows:

$$
H=\left\{\begin{array}{lc}
H_{1} & \sum_{i=1}^{M} K_{i} * \varphi_{i}>0 \\
H_{0} & \text { otherwise }
\end{array}\right.
$$

\section{SIMULATION RESULTS AND ANALYSIS}

To evaluate the performance of proposed techniques, Monte-Carlo simulations are carried out with 2,000 samples under following conditions: The number of CRSN node $M=6$, and these nodes are randomly located in a $100 \mathrm{~m} \times 100 \mathrm{~m}$ square area. The LU's signal is TV signal as in with $6 \mathrm{MHz}$ bandwidth, the local sensing time is $25 \mu \mathrm{s}$, and the identical number of samples $W$ is 300 . The sensing performance of the proposed scheme is compared with the sensing performances of the fuzzy logic based scheme [13] and the simple cooperative based scheme [12].

Firstly, the simulation was carried out under condition that the SNRS of LU's signal at nodes are $-15,-14,-13,-12$, -11 and $-14 \mathrm{~dB}$, respectively. Under such condition, the ROC curves of proposed scheme and comparison schemes are illustrated in Fig. (4). Fig. (4) shows that our scheme' performance is degradation as $a$ increases. Compared with the fuzzy logic based scheme, our scheme has a similar accuracy when $a=0.2$. When $a=0.6$, compared with the cooperative scheme, our scheme has a similar accuracy.
Secondly, our proposed scheme has been experienced under the condition that the SNRs of LU's signal at nodes are $-16,-14,-12,-8,-6$ and $-4 \mathrm{~dB}$, respectively. Under such condition, the error probability $P_{e}$ of our proposed scheme and comparison schemes is depicted on Fig. (5). For our proposed scheme, it can be seen that compared with the fuzzy logic based scheme, our scheme has a similar accuracy when $a=0.2$, but our scheme's error probability $P_{e}$ is smaller than the fuzzy logic scheme, when $S N R<-8 d B$. And we can see that $P_{e}$ increase as $a$ increases on Fig. (5).

Thirdly, our proposed scheme reduces the number of nodes in reporting and saves the energy of the CRSN nodes. In Fig. (6), when $a$ is increase, the number of reporting nodes will decrease. As seen in Fig. (6), when $a=0.2$, our proposed scheme only requires $60 \%$ of nodes to transmit their local decision to the fusion center. However, the optimal spectrum sensing scheme needs all nodes report their local decisions to the fusion center. When $a=0.5$, our proposed scheme only requires $20 \%$ of the number of nodes to report their local decision to the fusion center. It means that the proposed scheme can save the local decision to the fusion center in comparison with the conventional approach.

\section{CONCLUSION}

In this paper, a cooperative censoring spectrum sensing using the dependent function of extension theory for CRSN is proposed. A simple extension theory system is proposed to make the decision at each CRSN. Analysis and simulations show that the proposed scheme outperforms the cooperative scheme, and the proposed scheme keep the merits of the fuzzy logic scheme that reduce the number of nodes reporting 


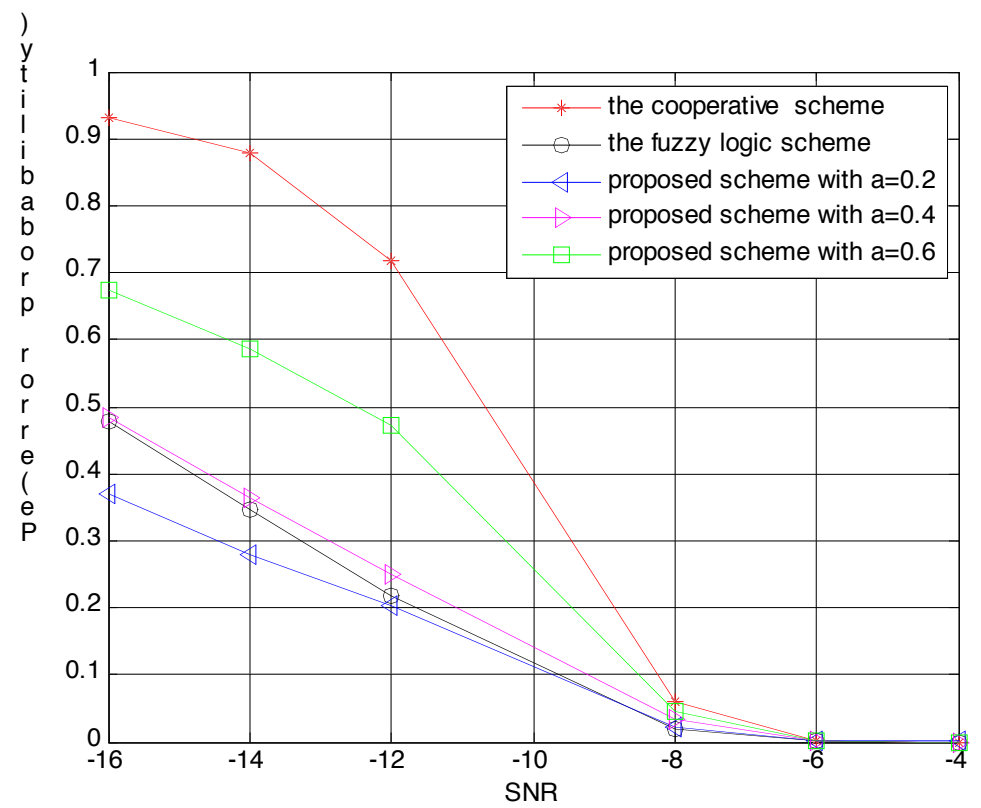

Fig. (5). The proposed scheme has been experienced under condition that the SNRs of LU'S signal at nodes are $-16,-14,-12,-8,-6$ and $-4 \mathrm{~dB}$, respectively. The comparison of the error probability be shown between proposed scheme and comparison schemes.

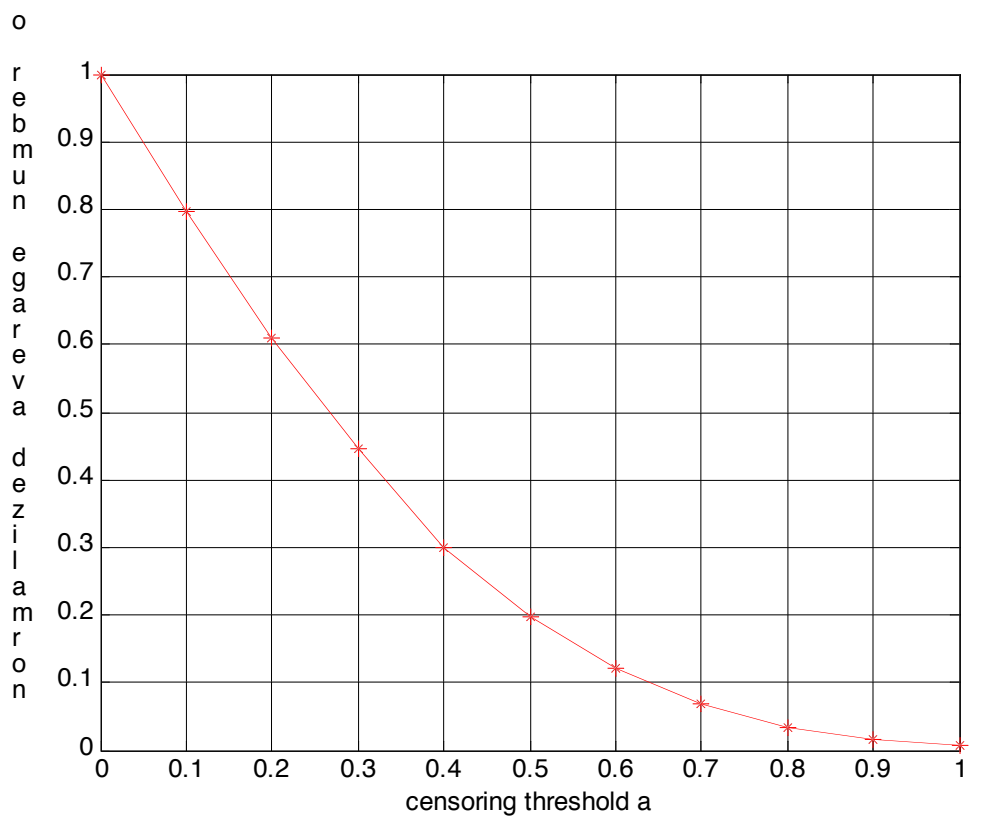

Fig. (6). When the censoring threshold under the condition that SNRs of LU's signal at nodes are $-15,-14,-13,-12,-11$ and $-14 \mathrm{~dB}$, respectively. The normalized average number of reporting nodes.

its local decision to the fusion center and save the energy of the CRSN nodes. When signal noise ratio of LU's signal at nodes is relatively small, the proposed scheme improves the detection probability effectively compared with the fuzzy logic scheme. For future work, more depth research will be done to satisfy the practical need.

\section{CONFLICT OF INTEREST}

The authors confirm that this article content has no conflict of interest.

\section{ACKNOWLEDGEMENTS}

This research was supported by The China Postdoctoral Science Foundation (No.2014M552529), Shenzhen New Industry Development Fund under grant (No.CXB201005250021A), the Developing Fund for Innovative Research Team of Guangdong University of Technology(No. GDUT2011-10), the Youth Foundation of Guangdong University of Technology (No. 13ZK0380) and the Ph.D. Programs Foundation of Guangdong University of Technology (No. 103042). 


\section{REFERENCES}

[1] F. Akyildiz, W. Su, Y. Sankarasubramaniam, and E. Cayirci, "A survey on sensor networks," IEEE Commun. Mag., vol. 40, no. 8, pp. 102-114, Aug. 2002.

[2] R. Ferri, M. J. Kim, and E. T. C. Yee, “Wireless Sensor Networks”, U. S. Patent 20,090,002,151, January 01, 2009.

[3] S. Abedi, “Wireless sensor networks”, U. S. Patent 8,310,359, Nov. 13, 2012.

[4] P. Juha, H., Anu, “Cognitive Radio”, U. S. Patent 20,120,129,462, May 24,2012.

[5] A. M. Barnawi, "Cognitive Radio Sensing Method and System”, U. S. Patent 20,110,263,208, Oct. 27, 2011.

[6] G. Memik, S. O. Memik, B. Mangione-smith, "Spectrum Sensing Network For Cognitive Radios", U. S. Patent. 20,110,026,376, Feb. 03, 2011.

[7] H. Tang, “Combined Sensing Methods For Cognitive Radio”, U. S. Patent. 20,140,018,008, Jan. 16, 2014

[8] K-C Fu, M-J Yang, and Y-F Chen, "Multistage wiener filter based spectrum sensing in cognitive radio", $12^{\text {th }}$ International Conference on ITS Telecommunications (ITST), 5-8, Nov. 2012, pp. 700-705.

[9] S. L. Sabat, S. Srinu, A. Raveendranadh, and S. K. Udgata, "Spectrum sensing based on entropy estimation using cyclostationary features for Cognitive radio," $4^{\text {th }}$ International Conference on Communication Systems and Networks (COMSNETS), 3-7, Jan. 2012, pp. 1-6.
[10] M. López-Benítez, and F. Casadevall, "Improved energy detection spectrum sensing for cognitive radio", IET Communications, vol. 6, no. 8 , pp. 785-796. May 2012.

[11] C. You, H. Kwon, and J. Heo, "Cooperative TV spectrum sensing in cognitive radio for Wi-Fi networks", IEEE Trans. Consumer Electron., vol.57, no. 1, pp. 62-67, Feb. 2011.

[12] F. Peng, H. Chen, and B. Chen, "On energy detection for cooperative spectrum sensing", $46^{\text {th }}$ Annual Conference on Information Sciences and Systems (CISS), 21-23, March 2012, pp. 1-6.

[13] T. Kieu-Xuan, and I. Koo, “A Censor-Based Cooperative Spectrum Sensing Scheme Using Fuzzy Logic for Cognitive Radio Sensor Networks", IEICE Trans. Commun., vol. E93B, no. 12, pp. 34973500, Dec. 2010.

[14] W. Cai, "Extension Theory and Its Application", Chin. Sci. Bull., vol. 44, no. 17, pp. 1538-1548, Sep. 1999.

[15] Y-H. Chen, Y-Q Yu, B. Zeng, "Extension detecting technology and the implementing method for its application in engineering" International Conference on Machine Learning and Cybernetics, 12-15 July 2009, vol. 2, pp. 631-636.

[16] H. Urkowitz, "Energy detection of unknown deterministic signals" Proceedings of the IEEE, April 1967, vol. 55, no. 4, pp. 523-531.

[17] V.I. Kostylev, "Energy detection of a signal with random amplitude" IEEE International Conference on Communications, 2002. ICC 2002, 2002, vol. 3, pp. 1606-1610.

[18] A.U. Makarfi, and K.A. Hamdi, "Interference analysis of energy detection for spectrum sensing”, IEEE Trans. Veh. Technol., July 2013, vol. 62 , no. 6 , pp. $2570-2578$.

(C) Ouyang et al.; Licensee Bentham Open.

This is an open access article licensed under the terms of the Creative Commons Attribution Non-Commercial License (http://creativecommons.org/licenses/by-nc/3.0/) which permits unrestricted, non-commercial use, distribution and reproduction in any medium, provided the work is properly cited. 